\title{
A very important little problem
}

This issue contains papers read at the 1996 Electronic Access to Fiction research seminar in Copenhagen by courtesy of Hanne Albrechtsen who organised the seminar at the Royal School of Librarianship.

The issue includes Inga-Lill Ekvall and Sibyl Larsson's piece about user's needs when asking about a certain kind of book in a library. It is full of common sense. The benefits of having only two people organising the indexing receives the author's attention: "It is good not to involve too many persons because the more persons involved the greater the risk that similar books will be treated differently".

This reminds me of an experiment conducted by Broadbent and Broadbent reported in the journal Ergonomics back in the days when PC meant Police Constable (1978). They concluded "People retrieve desired material better when they use descriptors they themselves have assigned to an item".

Today additional bells and whistles are the thing. You will rarely come across a new product where indexing - today almost as rare as Police Constables and at least as important - is featured. As a matter of fact indexing is not a little problem at all; it is more important then it ever was. Ekvall et al. could have mentioned another severe indexing problem - the time it takes to do it properly.

The records in my office database form the index to a large collection of documents. Indexing is not directly productive, so the name of the game is to reduce the time you spend on indexing to an absolute minimum. The guideline is Cawkell's first law: "Thou shalt do only that amount of indexing which results in a just satisfactory retrieval performance". Unfortunately retrieval performance is so hard to measure that its decline is only noticed when stuff which you know is there is not retrieved. Even so, indexing is so costly in time that it deserves the crude attention just described - at the very minimum.

A.E. Cawkell 